\title{
Macroscopic Modeling of Water Uptake Behavior of PEDOT:PSS ${ }_{2}$ Films
}

\author{
3 Lyazzat Zhanshayeva, Valentina Favaron, and Gilles Lubineau*(i) \\ 4 Division of Physical Sciences and Engineering, COHMAS Laboratory, King Abdullah University of Science and Technology \\ 5 (KAUST), Thuwal 23955-6900, Saudi Arabia
}

6

Supporting Information

7 ABSTRACT: Poly(3,4-ethylene dioxythiophene)-poly(styrene sulfo-

8 nate) (PEDOT:PSS) is a widely used conductive polymer (CP) for applications in electronic devices. In the context of transparent electrodes or soft actuators, the uptake of water by PEDOT:PSS is an essential element in the performance of the physical system. We study the water uptake of pure films and films treated with ethylene glycol (EG) commonly used to enhance the electrical properties of PEDOT:PSS. Gravimetric analysis was used to investigate the water sorption-desorption of PEDOT:PSS and its change with the EG treatment for a wide range of configurations (thickness, temperature, and relative humidity). We demonstrate that a simple Fickian model cannot correctly represent the experimental results; we therefore introduce a fully coupled reaction-diffusion scheme. This model

Modeling for accurate simulation of water update in PEDOT/PSS for: - different thicknesses - different solvent pre-and post- treatments, - different environments - both sorption and desorption Environment
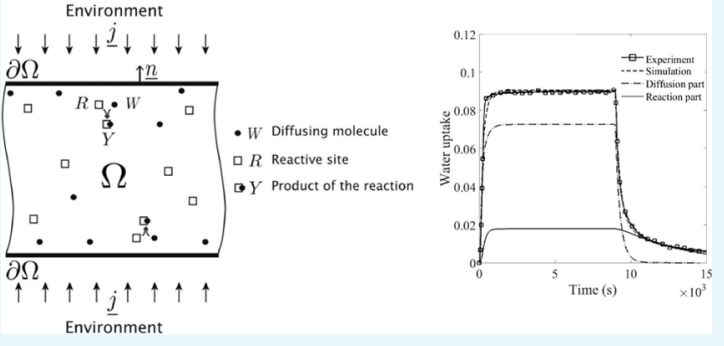
Environment describes the transport of diffusing molecules into the polymer film, taking into account, in addition to the classical diffusion mechanism, the reaction between the reactive sites of the polymer network and the water molecules. We demonstrate that solvent treatments have a direct influence on the kinetics of the water uptake of PEDOT:PSS films in terms of diffusivity, solubility, and rate of reaction. The proposed model can be used to accurately predict the water uptake of $\mathrm{CP}$ films in the case of complex three-dimensional configurations that are needed for the design of complex actuators.

\section{INTRODUCTION}

27 Because of their lightweight, flexibility, and ease of 28 processability, organic conductive polymers $(\mathrm{CPs})$ are widely 29 applied in electronic devices. ${ }^{1}$ The most successful polymer is 30 poly(3,4-ethylenedioxythiophene)-poly(styrene sulfonate) 31 (PEDOT:PSS). Its tunable electrical properties (conductivity 32 can reach $4000 \mathrm{~S} / \mathrm{cm}$ by using proper treatment ${ }^{2-4}$ ), low33 voltage-driven actuation mechanism, ${ }^{5}$ and the recently revealed 34 self-healing effect ${ }^{6}$ make PEDOT:PSS a good candidate for 35 transparent electrodes, organic solar cells, organic light36 emitting diodes, wearable textiles, artificial muscles, sensors, 37 and actuators. ${ }^{1,5,7-10}$

38 PEDOT:PSS features a strong coupling among its chemical, 39 mechanical, and electrical behaviors, which has resulted in 40 many applications in the field of electromechanical actuators. ${ }^{11}$ 41 The mechanism of actuation comes from the hygroscopic 42 nature of PSS that very easily absorbs water from the 43 environment. Water molecules can actually exist in very 44 different configurations. As shown by ref 12 , based on the 45 observation of isosteric heat of sorption, water molecules will 46 mainly be adsorbed on the hydrophilic sulfonic acid groups at a 47 low sorption degree. At a higher sorption degree, the active 48 sites are totally saturated and water is in a free-water 49 configuration. The absorbed water molecules increase the so distance between the PEDOT:PSS grains, causing a volume increase. When an electric current is applied, the Joule effect 51 results in the generation of in-the-volume heat, consequently 52 changing the temperature. It results in the release of the 53 previously absorbed water molecules, which translates into 54 geometrical shrinking at the macroscale. PEDOT:PSS-based 55 actuators can be operated under low voltages $(0-10 \mathrm{~V})$ that 56 makes them particularly appealing for wearable electronic 57 applications. ${ }^{5}$

Considering the phenomenology described above, water 59 content plays a major role in PEDOT:PSS actuation 60 properties. This phenomenology has been well studied for 61 other families of CPs such as polypyrrole, with the pioneering 62 work of Okuzaki's group, ${ }^{13-15}$ but still additional data are 63 needed in case of PEDOT:PSS. More generally, all properties 64 of PEDOT:PSS are highly dependent on its water content. A 65 decrease in Young's modulus, along with an increase in the 66 relative humidity $(\mathrm{RH})$ of the environment, was reported in 67 previous studies. ${ }^{16}$ This happens because of the weakened 68 hydrogen bonding between the PEDOT and PSS grains, 69 during the water uptake of hygroscopic PSS. In addition, water 70 molecules increase the distance between PEDOT oligomers, 71

Received: September 4, 2019

Accepted: November 15, 2019 
72 resulting in an increase of electrical resistivity. ${ }^{17} \mathrm{~A}$ change in 73 the distribution of both stress and strain was also observed. ${ }^{16}$ 74 These results illustrate the crucial role of the water sorption 75 mechanism of PEDOT:PSS in modifying the complete 76 behavior of the film. Today, the phenomenological aspects of 77 the PEDOT:PSS actuation and water sorption are well known. 78 The amount of water vapor sorption decreases with an increase 79 in temperature and hysteresis between the sorption and 80 desorption isotherms that were previously reported, ${ }^{11}$ and 81 there is a good understanding of the sorption isotherms from 82 the thermodynamic viewpoint. Interesting works have been 83 done recently, investigating the effect of microstructure (and 84 especially the PEDOT:PSS ratio) on the coupling among water 85 sorption, swelling, and electrical properties. ${ }^{18}$ However, to the 86 best of our knowledge, there is no model or systematic data to 87 describe the interplay among water absorption, mechanical 88 expansion, and electrical stimulus. Elucidating the role of each 89 component in the actuation properties is needed to further 90 optimize and tailor soft actuators based on such materials.

91 The objective of this paper is to provide a reliable 92 macroscopic modeling of the water uptake in PEDOT:PSS 93 films that can be used to better design transparent 94 PEDOT:PSS electronics or actuators. To do so, we explore a 95 wide range of experimental conditions, both during the 96 sorption and desorption tests in order for our set of data to 97 discriminate between the different modeling approaches.

98 We first used gravimetric analysis to understand the 99 absorption-desorption mechanism by monitoring the water 100 uptake of the film at a macroscopic level. The obtained data 101 clearly show that a simple Fickian model presents severe 102 limitations; we therefore need a more complex modeling 103 approach. We decide to adopt the reaction-diffusion scheme 104 proposed by El Yagoubi et al. ${ }^{19}$ to model the water uptake 105 behavior. This model takes into account the reaction between 106 water molecules and the substrate, in addition to the classical 107 diffusion. Finally, we discuss how the constitutive parameters 108 of this model are modified when classical treatments, such as 109 pretreatment or post-treatment with ethylene glycol (EG), are 110 used.

\section{MATERIALS AND METHODS}

112 Material Preparation. An aqueous PEDOT:PSS dis113 persion (Clevios PH1000) was purchased from HC Starck, 114 Inc. PH1000 was chosen because of its high electrical 115 conductivity in comparison to other commercially available 116 grades. It has a $1: 2.5 \mathrm{w} / \mathrm{w}$ ratio of PEDOT to PSS. We study 117 three types of films: pure, pretreated, and post-treated 118 PEDOT:PSS. For each configuration, a drop-casting technique 119 was used to prepare the films. A square Petri dish of size $8.5 \mathrm{~cm}$ $120 \times 8.5 \mathrm{~cm}$, covered with a Teflon paper, was plasma-treated to 121 remove the impurities and to maximize wettability. An 122 Expanded Plasma Cleaner PDC-001 from Harrick Plasma 123 was used at high power and $400 \mathrm{mTorr}$ pressure, for $5 \mathrm{~min}$. An 124 adequate amount of the dispersion was then poured into the 125 dish and cured in a fume hood for $48 \mathrm{~h}$, at room temperature. 126 The pretreatment process was performed by adding the 127 solvent EG (3 wt \%) to the aqueous PEDOT:PSS dispersion. 128 The solution was mixed for $6 \mathrm{~h}$ in a fume hood.

129 The post-treatment process was performed on a pure 130 PEDOT:PSS film. The pure PEDOT:PSS film samples were 131 placed inside a beaker filled with EG for $6 \mathrm{~h}$ and then removed. 132 The pretreated and post-treated films were annealed at 120 $133{ }^{\circ} \mathrm{C}$ for $2 \mathrm{~h}$. Let us note that we use "pretreated" and "post- treated" for naming samples after EG addition or EG rinsing 134 and that we do not refer to oxidation/reduction of the CP. 135 Three sample thicknesses $(2 L)$ were considered for pure films: 136 $2 L=15 \mu \mathrm{m}, 2 L=50 \mu \mathrm{m}$, and $2 L=100 \mu \mathrm{m}$, whereas only 50137 $\mu \mathrm{m}$ was used for pretreated and post-treated films. The choice 138 of relatively large thicknesses is motivated by applications on 139 soft robotics and soft actuators, for which thicknesses are 140 usually much larger compared to the films used for transparent 141 electrodes. Film thicknesses were measured using a digital 142 micrometer with a $1 \mu \mathrm{m}$ accuracy. The drop-casting method is 143 known for the ease of film preparation, but this method makes 144 it difficult to control the film thickness and uniformity. 145 Therefore, there was a $10 \%$ variation of thickness in polymer 146 film samples. For this reason, the thickness of each individual 147 sample was measured after processing, in order to secure 148 accurate data.

Gravimetric Test. For gravimetric measurements, dried 150 polymer films were cut into $7 \mathrm{~mm}$ by $7 \mathrm{~mm}$ squares using a 151 $\mathrm{CO}_{2}$ universal laser system PLS 6.75. The following laser 152 settings were used: speed: $5.0 \%$; pulses per inch: 750; $Z$-axis: 153 $4.00 \mathrm{~mm}$; and power: $0.5,1.5$, and $2 \%$, depending on the 154 thickness of the film.

An IGAsorp dynamic vapor sorption (DVS) analyzer 156 (Hiden Isochema) was used for gravimetric measurements. 157 The temperature and $\mathrm{RH}$, within the isolated chamber, were 158 controlled while monitoring the mass uptake of the specimen 159 with an embedded $10 \mu \mathrm{g}$ resolution microbalance. The 160 polymer samples were placed on a suspended stainless steel 161 mesh inside the chamber, in order for both sides of the film to 162 be exposed to the environment. Three configurations of the 163 material were considered: pure, pretreated, and post-treated 164 PEDOT:PSS. For each configuration, different samples were 165 tested at different temperatures $\left(26,37,50\right.$, and $\left.80{ }^{\circ} \mathrm{C}\right)$ and 166 RH's (30, 60, and 80\%), making a total of 36 different 167 configurations. For each configuration, both the absorption 168 and desorption responses were investigated. Each test was 169 repeated three times.

We performed a systematic conditioning of the film: the 171 water content of the film was removed prior to humidity 172 exposure by drying the sample inside the equipment until the 173 mass equilibrium was reached. The dried polymer films were 174 exposed to a flow of $250 \mathrm{~mL} / \mathrm{min}$ moist air until stabilization, 175 followed by a desorption test, by keeping the temperature 176 constant but prescribing the humidity level to $0 \%$. The gas 177 environment was controlled by mixing wet air with a dry flow 178 of nitrogen. The DVS analyzer allowed the measurement of the 179 mass uptake, $m(t)$, every $20 \mathrm{~s}$, which is a relatively high rate. 180

The results from the gravimetric tests were plotted as a ratio 181 of the mass of absorbed water by the PEDOT:PSS film, $\Delta m$, to 182 the mass of the dry sample, $m_{0}$, defined as relative water uptake 183

$$
m_{\exp }=\frac{\Delta m}{m_{0}}
$$

where

$$
\Delta m=m-m_{0}
$$

Numerical Implementation. Gravimetric tests were 187 reproduced using the COMSOL Multiphysics 5.2 software in 188 which we implemented the model described briefly in the 189 section and completely in the Supporting Information. 190 Assuming that the thickness of the polymer film is much less 191 than its width and length, a one-dimensional simulation was 192 


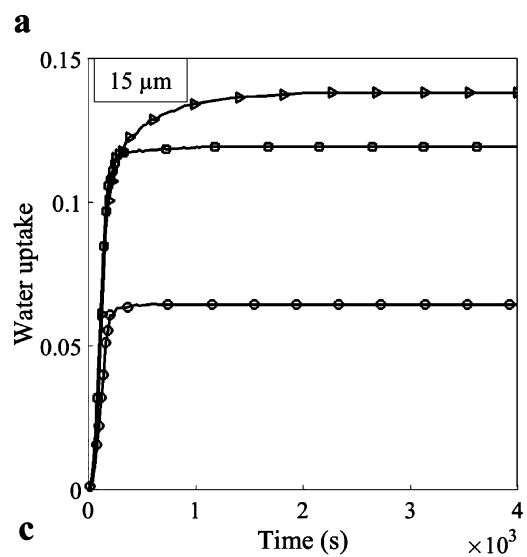

b
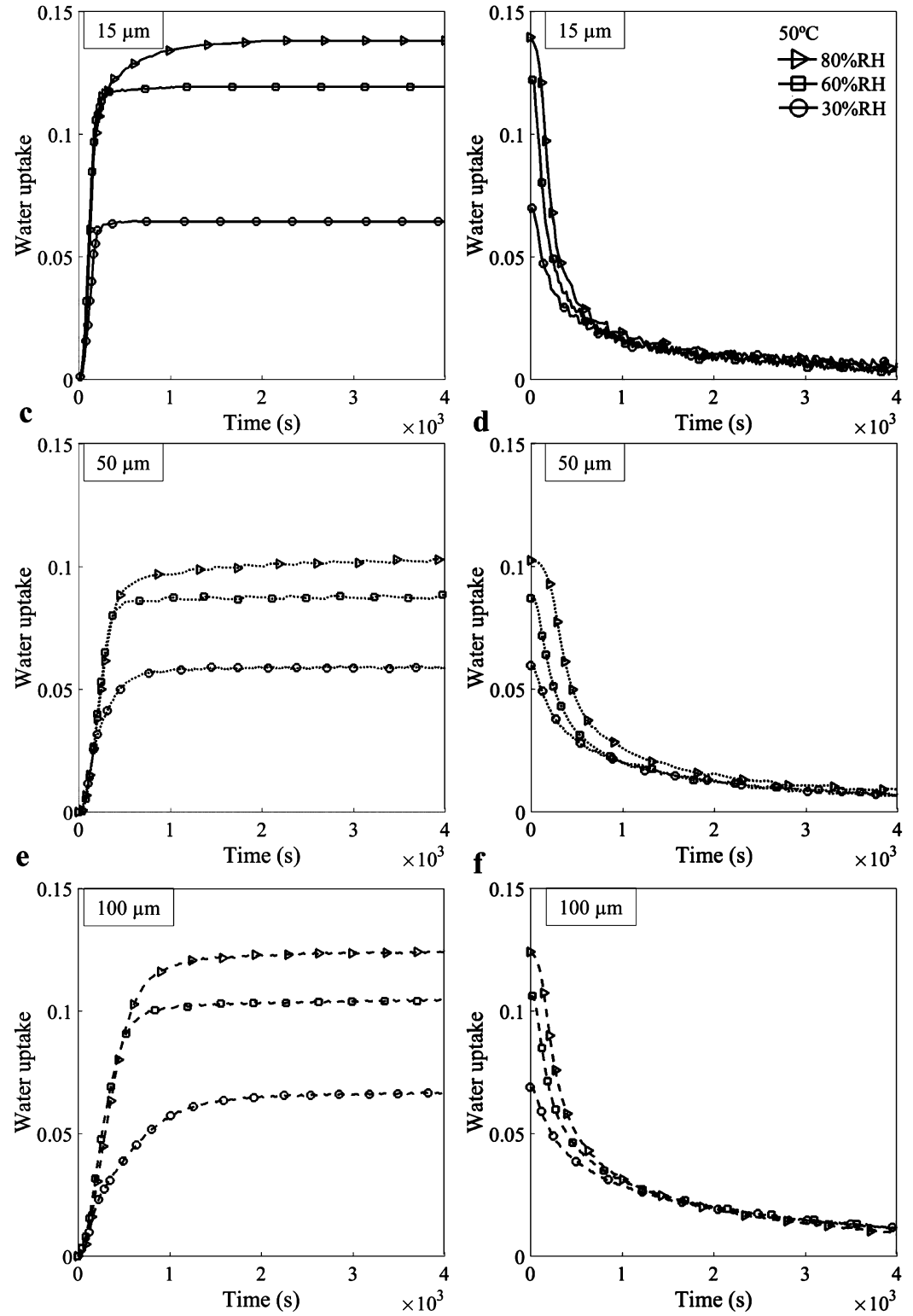

Figure 1. Sorption-desorption curves for $(\mathrm{a}, \mathrm{b})$ 15, (c,d) 50, and (e,f) $100 \mu \mathrm{m}$ thick pure PEDOT:PSS films exposed to 30\% RH, 60\% RH, and $80 \% \mathrm{RH}$ at $50{ }^{\circ} \mathrm{C}$. The water uptake is dimensionless and defined by eq 1 .

193 conducted. Considering the symmetry of the film, half of the 194 thickness $(L)$ was analyzed. The RH values were taken directly 195 from the results file of the DVS chamber, for all configurations, 196 to reproduce a real environment.

197 The mass uptake was derived, considering both the free $(w)$ 198 and bonded water $(Y)$

199

$$
m_{\text {sim }}=\frac{\Delta m}{m_{0}}=\frac{M_{\mathrm{H}_{2} \mathrm{O}} \int_{\Omega}(w+Y) \mathrm{d} V}{\rho_{0} V_{0}}
$$

200 where $M_{\mathrm{H}_{2} \mathrm{O}}=0.018 \mathrm{~kg} / \mathrm{mol}$ is the molar mass of water, $\rho_{0}=$ $2011350 \mathrm{~kg} \mathrm{~m}^{-3}$ is the initial density, and $V_{0}$ is the initial volume 202 of the sample.

203 The simulation and experimental curves were compared 204 using a MATLAB Optimization Toolbox function "lsqnonlin", 205 which solves nonlinear least-squares data-fitting problems. The 206 seven parameters of the model $\left(S_{0}, S_{1}, D_{0}, D_{1}, k_{\mathrm{h}}, k_{\mathrm{r}}\right.$, and $R_{0}$; 207 see Supporting Information) were optimized. "Isqnonlin" minimizes the sum of squares of the vector-valued function, 208 which is the difference between the experimental and 209 simulation data. Also, the initial value of the function and its 210 lower and upper bounds were specified individually, for each 211 parameter. Iteration terminates when the solver reaches the 212 stopping criteria specified by the user. For this problem, 213 stopping criteria were the tolerance of the function value $(1 \times 214$ $10^{-8}$ ) and the number of maximum iterations (500). Each 215 curve was optimized separately, and the parameters found for 216 the first simulated curve were used as initial values for the next 217 curves.

\section{RESULTS}

219

Here, we only discuss raw experimental results without 220 introducing any modeling consideration or opinion, which 221 we will do in the Discussion section.

Figure 1 shows the evolution of the relative water uptake of $223 \mathrm{fl}$ pure PEDOT:PSS as a function of \% RH change, at a constant 224 

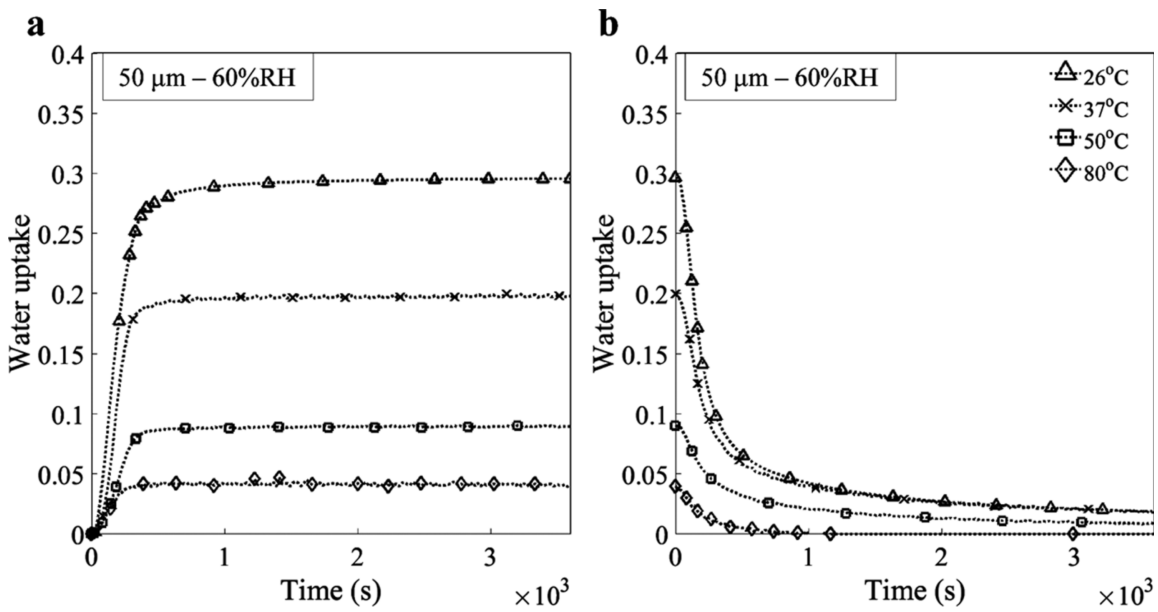

Figure 2. (a) Sorption and (b) desorption curves for a $50 \mu \mathrm{m}$ pure PEDOT:PSS film exposed to $60 \% \mathrm{RH}$ at $26,37,50$, and $80{ }^{\circ} \mathrm{C}$.
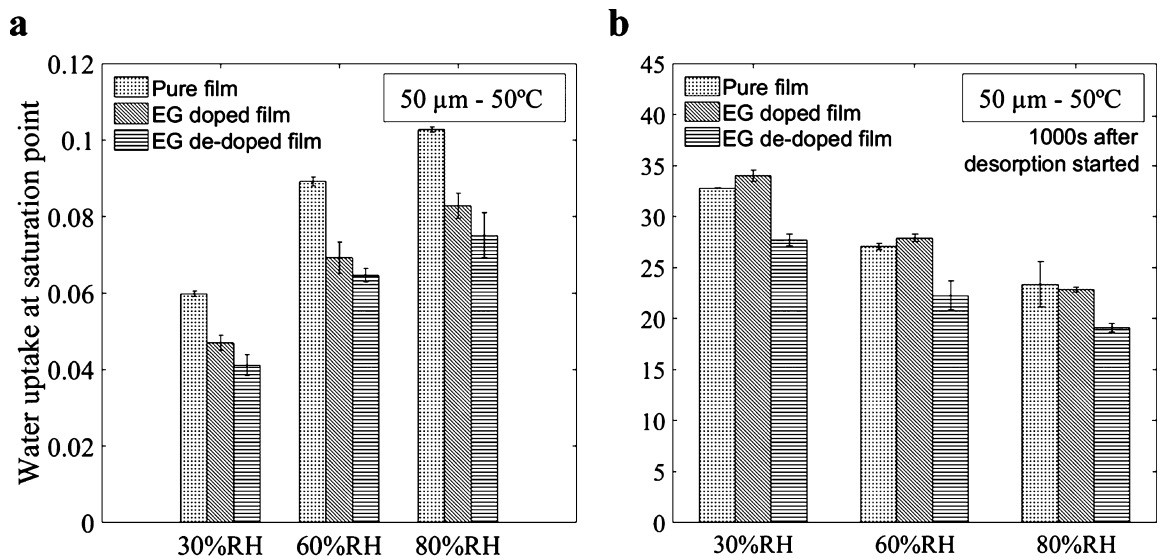

Figure 3. (a) Water uptake of a $50 \mu \mathrm{m}$ thick pure, pretreated, and post-treated PEDOT:PSS film exposed to $30 \% \mathrm{RH}, 60 \% \mathrm{RH}$, and $80 \% \mathrm{RH}$ at a saturation point. (b) Percent of water content after $1000 \mathrm{~s}$ of desorption started at $50{ }^{\circ} \mathrm{C}$.

225 temperature of $50{ }^{\circ} \mathrm{C}$, for three thicknesses: (a,b) 15 , (c,d) 50 , 226 and (e,f) $100 \mu \mathrm{m}$. The corresponding data for other 227 temperatures are available in the Supporting Information 228 (Figures S4-S7). Gravimetric experiments were found 229 reproducible for all configurations. One test for each case is 230 presented. Stabilization of mass was reached for all 231 experimental configurations during the $3 \mathrm{~h}$ absorption test. 232 We only plot the first hour of water absorption and the first 233 hour of water desorption. The amount of absorbed water varies 234 from a minimum of $3 \%\left(30 \% \mathrm{RH}, 80{ }^{\circ} \mathrm{C}\right.$, see Figure $\left.\mathrm{S} 7\right)$ to a 235 maximum of $56 \%\left(80 \% \mathrm{RH}, 26{ }^{\circ} \mathrm{C}\right)$. All sorption curves 236 (Figure 1a,c,d) display a saturation plateau that is constant 237 until desorption starts.

238 It is important to note the asymmetry between the 239 absorption and desorption processes. During the absorption 240 process, the polymer film uptakes water molecules relatively 241 fast at the beginning, but the desorption process appears much 242 slower. After a $1 \mathrm{~h}$ desorption process, we found that the 243 PEDOT:PSS films still contained some residual mass uptake. 244 We also observed that higher humidity level slightly increases 245 the amount of residual water in the network.
246 Figure 2 illustrates the sorption-desorption curves for a 50 $247 \mu \mathrm{m}$ pure PEDOT:PSS film exposed to $60 \% \mathrm{RH}$ at four 248 different temperatures. The amount of diffused water decreases 249 with an increase in temperature. This result is consistent with 250 Okuzaki's findings. ${ }^{11}$ The saturation point is reached faster at higher temperatures, both for sorption (Figure 2a) and 251 desorption (Figure 2b).

Figure 3a presents how the solvent treatment affects the $253 \mathrm{f} 3$ water uptake process. We still do not introduce any model here 254 and only focus on facts that can be directly observed from the 255 experimental curves. The results indicate that the EG 256 treatment (both pretreatment and post-treatment) induces a 257 decrease of water uptake in the film, at saturation, in 258 comparison with pure films, because of the structural changes 259 it causes. The water uptake of the pretreated film is also always 260 higher than the one of the post-treated film. This is consistent 261 with the removal of PSS sites from the surface of the 262 PEDOT:PSS film during the post-treatment process, which 263 results in a decrease in the amount of reactive sites that bond 264 with water molecules. In addition, the normalized water 265 content of the post-treated film after $1000 \mathrm{~s}$ of desorption is 266 less than that of pure and pretreated films (Figure 3b). A 267 slightly faster water release is due to the lower number of 268 reactions taking place in the polymer network associated with 269 less PSS. It means that the part of free volume-associated water 270 is more important in post-treated films than in other films, 271 which is also consistent with the removal of PSS by washing. 272 We would ascribe the slower desorption in the pretreated film 273 compared to pure films to a reorganization of the micro- 274 structure (at constant PSS content). Because of the changes in 275 conformation in the chains, the microstructure rearranges, 276 

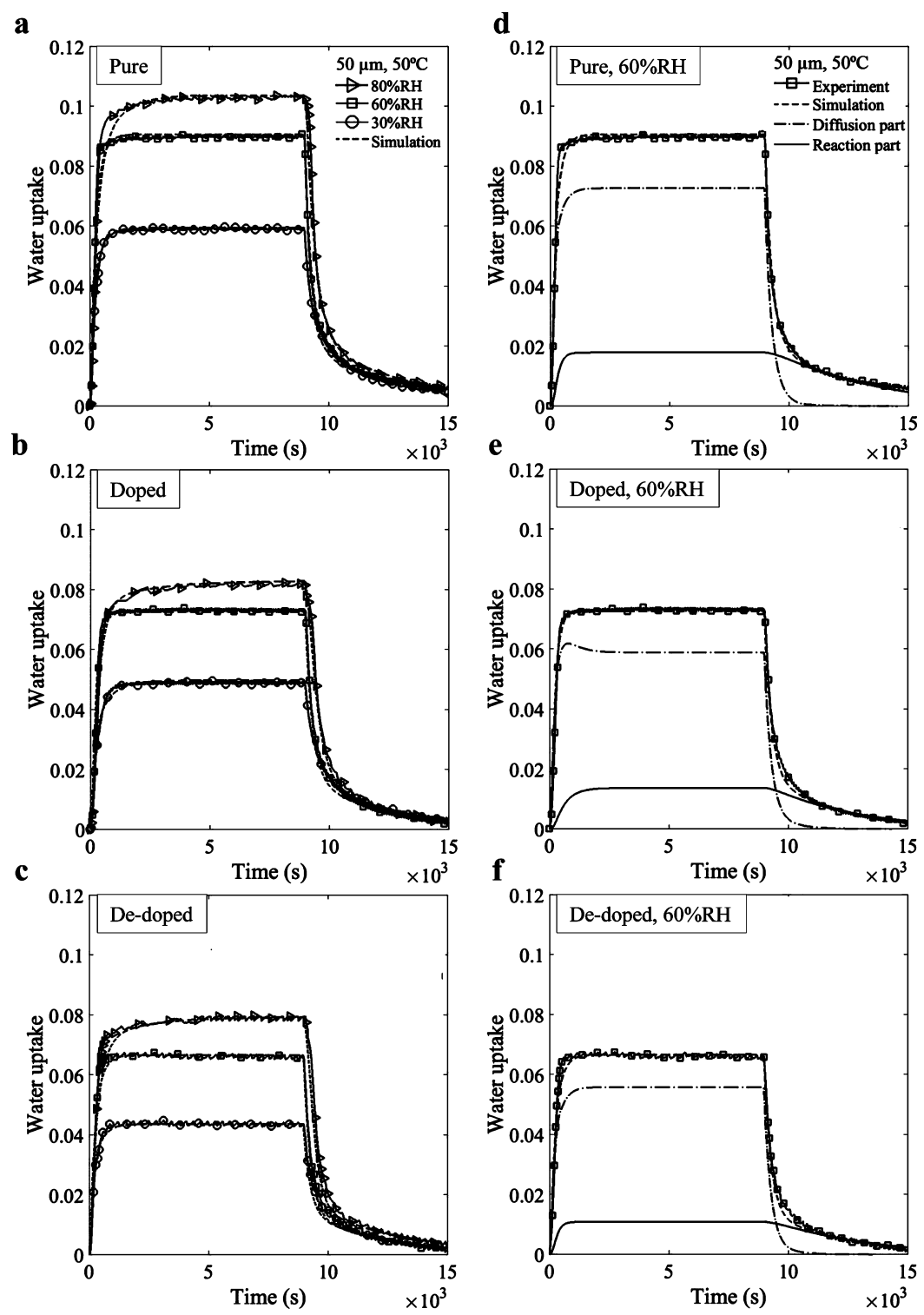

Figure 4. $(\mathrm{a}-\mathrm{c})$ Comparison between the simulation and experimental curves for $50 \mu \mathrm{m}$ at $50{ }^{\circ} \mathrm{C}$; $(\mathrm{d}-\mathrm{f})$ contribution of "diffusing" and "reacting" water into the water uptake of PEDOT:PSS exposed to $60 \% \mathrm{RH}$.

277 resulting in a more dense network, less free volume, and slower 278 desorption. This effect is, however, of second order as the 279 associated variations are very small.

\section{DISCUSSION}

281 These experimental results (asymmetry in kinetics between 282 absorption and desorption and the existence of a non283 negligible amount of residual water uptake after drying) 284 suggest a non-Fickian absorption behavior. Indeed, we tried to 285 represent these experimental results using a simple Fickian 286 approach, but we could not represent both absorption and 287 desorption with a unique set of material parameters. An 288 example is provided in the Supporting Information (Section 289 1). Our results suggest a much more complex response that 290 should be taken into account for the interactions between the 291 diffused water and the substrate. The change in the 292 microstructure triggered by the EG treatment can also strongly 293 influence this interaction.

294 We adapt a more general model introduced by El Yagoubi 295 and Lubineau. ${ }^{19}$ In addition to the classical diffusion, it takes into account the reaction of water molecules with the substrate 296 that result in a global diffusion-reaction mechanism. The 297 diffusing molecules, $w$, and the reactive site of the polymer, $R, 298$ form a complex $Y$

$$
R+w \Leftrightarrow Y
$$

where $w, R$, and $Y$ coexist in the domain $\Omega$. The unit of 301 concentration is $\mathrm{mol} \mathrm{m}^{-3}$.

We assume a first-order reaction for which the global rate of 303 creation/consumption of water $\left(r_{\mathrm{w}}\right)$ is given by the 304 concentrations of reactants and products and by the rate 305 constants $\left(k_{\mathrm{h}}\right.$ : rate constant of the forward reaction, $k_{\mathrm{r}}$ : rate 306 constant of the backward reaction) in a constant volume $\quad 307$

$$
r_{\mathrm{w}}=-k_{\mathrm{h}}(T) w R+k_{\mathrm{r}}(T) Y
$$

where $k_{\mathrm{h}}$ in $\mathrm{m}^{3} \mathrm{~mol}^{-1} \mathrm{~s}^{-1}$ and $k_{\mathrm{r}}$ in $\mathrm{s}^{-1}$ are the functions of 309 temperature.

The change of water concentration, at any point and any 311 time, is the result of both the diffusion of free water and its 312 
313 interaction with the substrate (to create the complex $Y$ made of 314 the substrate and bonded water), such that

$$
315 \quad \frac{\partial w}{\partial t}=-\operatorname{div}(\underline{j})+r_{\mathrm{w}}
$$

316 where $j$ is the free-water flux. The full model is described in the 317 Supporting Information (Section 2). The free-water flux (and 318 diffusion of water in general) mainly depends on two material 319 parameters: the diffusivity $D\left(\right.$ in $\mathrm{m}^{2} \mathrm{~s}^{-1}$ ) and the solubility $S$ 320 (in $\mathrm{mol} \mathrm{m}^{-3} \mathrm{~Pa}^{-1}$ ). In case of non-Fickian diffusion, these 321 material parameters eventually largely depend on the micro322 structure that evolves in time because of the reaction. Indeed, 323 any structural reorganization will modify the free volume and 324 the internal diffusion path, eventually modifying the effective 325 macroscopic diffusion coefficient and solubility that measure 326 how fast the diffusion is and how much can be absorbed, 327 respectively. We model the effects of these microstructural 328 changes in a simple way. From a macroscopic perspective, we 329 assume a linear relationship among the reaction product $Y$, the 330 diffusivity $D$, and/or the solubility $S$

$$
331 \quad D(Y)=D_{0}+D_{1} Y ; S(Y)=S_{0}+S_{1} Y
$$

332 As $Y$ is the product of the reaction, with an initial 333 concentration equal to zero, a linear relationship is the most 334 simple model we can have that comes naturally from an 335 asymptotic expansion around zero. Of course, more complex 336 relations could be imagined, but these would not be relevant 337 here as we could not discriminate between them based on the 338 available experimental data. To summarize, this model 339 describes the progressive conversion of the reactive sites $(R)$ 340 of the substrate into bonded water $(Y)$ by interaction with the 341 diffusing water $(w)$. The initial concentration of the reactive 342 site available in the polymer film to undergo chemical reaction 343 is noted $R_{0}$. The complete formulation of the model, for our 344 case, and the numerical implementation in COMSOL 345 Multiphysics and MATLAB Optimization toolbox, are given 346 in the Supporting Information.

347 After a necessary calibration step (described in the 348 Supporting Information), we first demonstrated that this 349 diffusion-reaction model was able to describe the absorption350 desorption results for pure, pretreated, and post-treated films at 351 all $(T, \% \mathrm{RH})$ configurations. The data for the $50 \mu \mathrm{m}$ film 352 exposed to three humidity levels at $50{ }^{\circ} \mathrm{C}$ are presented in 353 Figure $4 \mathrm{a}-\mathrm{c}$, where dashed lines represent the corresponding 354 simulation results. Figure $4 \mathrm{~d}-\mathrm{f}$ shows the contribution of 355 "diffusing" $(w)$ and "reacting" $(Y)$ water into the water uptake 356 of pure, pretreated, and post-treated PEDOT:PSS films at 50 $357{ }^{\circ} \mathrm{C}$ exposed to $60 \% \mathrm{RH}$. According to these results, mass 358 uptake comes mainly from the diffusion of free water, but the 359 bonded water described in the "reaction part" largely 360 contributes to explain the response during desorption. The 361 proposed model described all configurations with similar 362 accuracies.

363 It is then interesting to study and explain how the 364 parameters of the model vary with the different configurations. 365 The initial solubility constant $S_{0}$, the initial diffusivity constant $366 D_{0}$, and the quantity $R_{0}$ of the sulfonate groups accessible for 367 reaction at the beginning of the process are of particular 368 interest. At room temperature, and for pure PEDOT:PSS, their 369 values were $S_{0}=11.1 \mathrm{~mol} \mathrm{~m}^{-3} \mathrm{~Pa}^{-1}, D_{0}=1.6 \times 10^{-12} \mathrm{~m}^{2} \mathrm{~s}^{-1}$, 370 and $R_{0}=2730 \mathrm{~mol} \mathrm{~m}^{-3}$, respectively.

371 Figure 5 illustrates how the aforementioned parameters vary 372 with various solvent treatments and at different temperatures. a

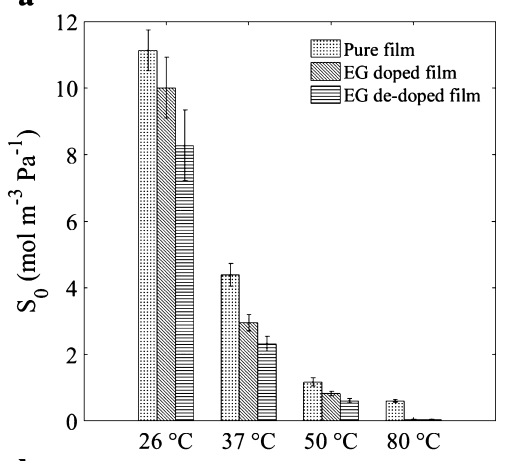

b
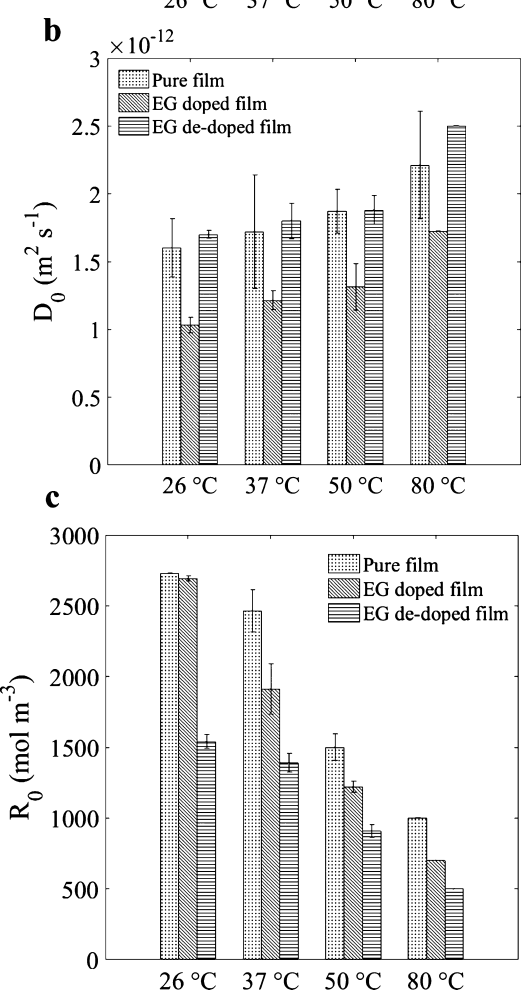

Figure 5. Evolution of (a) solubility constant $S_{0}$, (b) diffusion constant $D_{0}$, and (c) concentration of available reactive sites $R_{0}$ with temperature increase for pure, EG-treated, and post-treated films.

The way these parameters change, depending on the 373 configuration, provides additional insights into the physical 374 process and the following important parameters as discussed 375 below.

376

About the Solubility Constant $\boldsymbol{S}_{0}$ with Temperature. 377 In all cases, $S_{0}$ decreases with a temperature increase (Figure 378 5a). Higher temperatures lead to the strengthening of the 379 hydrogen bond between the PSS chain and the PEDOT:PSS 380 grains. ${ }^{20}$ This means that it will be more difficult for the water 381 molecules to bond with the sulfonate groups, as less space is 382 made available for the uptake of water.

383

About the Diffusivity Constant $D_{0}$ with Temperature. 384 $D_{0}$ increases with a temperature increase for all film types 385 (Figure 5b); however, the change is not drastic, compared to 386 $S_{0}$. This can be explained by two competing mechanisms: (i) 387 the increase in temperature causes an increase in the kinetic 388 energy of diffusing molecules, resulting in an acceleration of 389 the diffusion process (this mechanism usually explains the 390 exponential change with temperature of the diffusivity constant 391 for a pure Fickian process) and (ii) the drying polymer film 392 
$\mathbf{a}$

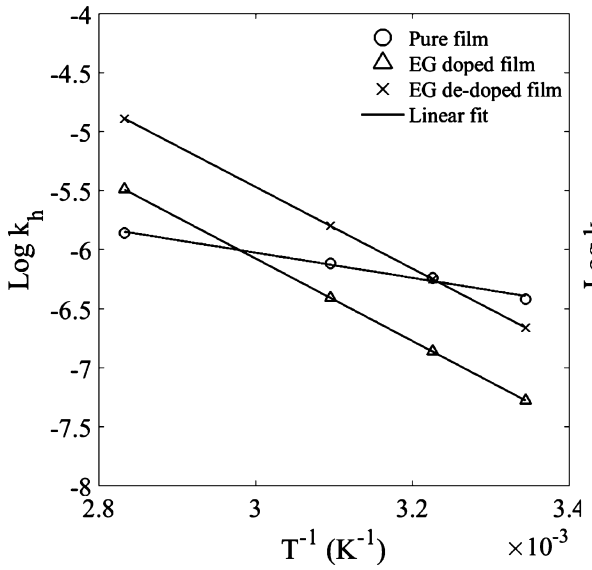

b



Figure 6. Change of rate constants (a) $k_{\mathrm{h}}$ and (b) $k_{\mathrm{r}}$ with temperature, for pure, EG-treated, and post-treated films.

393 becomes densely packed, decelerating the diffusion process. 394 The first phenomenon is dominant, as $D_{0}$ still has a positive 395 trend with the increase in temperature.

396 About the Quantity of Available Sites for Reaction $\boldsymbol{R}_{\mathbf{0}}$ 397 with Temperature. The decrease of the reactive substrate 398 concentration $\left(R_{0}\right)$ with temperature is also ascribed to the 399 change in the microstructure. Less PSS is accessible to undergo 400 a chemical reaction in the compacted structure, which 401 translates into a reduction of the apparent quantities of sites 402 available for reaction $\left(R_{0}\right)$. An in-depth investigation of the 403 change in the microstructure with temperature can be found in 404 ref 20

405 The observed evolution of these parameters with the 406 addition of solvents (Figure 5) is also compatible with the 407 expected changes in the PEDOT:PSS film's structural order as 408 discussed below.

409 About the Evolution with EG Pretreatment. Adding an 410 EG solvent into the PEDOT:PSS aqueous solution changes the 411 conformation of the PEDOT chains from a benzoid (coiled) to 412 a quinoid structure (linear). ${ }^{21}$ This leads to an increase in the 413 number of interchain interactions because interactions among 414 chains of linear conformation are facilitated, compared to the 415 coiled conformation. Therefore, EG enhances the organization 416 of the microstructure and promotes a more packed 417 configuration. ${ }^{22,23}$ Such a microstructure will be more difficult 418 to penetrate and will leave less free volume for the diffusion 419 process to happen. These changes can be seen in Figure 5a, 420 where the solubility of the pretreated film is lower than the 421 solubility of the pure film; it results in the slow diffusion 422 process $\left(D_{0}\right.$ decreased, see Figure $\left.5 \mathrm{~b}\right)$ and in the reduction of 423 concentration of accessible sulfonate groups for bonding (see 424 Figure 5c).

425 About the Evolution with EG Post-Treatment. The 426 pure PEDOT:PSS film was post-treated by immersion into the 427 EG solvent, which partially removes the hygroscopic PSS from 428 the surface. Therefore, it uptakes the least amount of water 429 among the three films (Figure 5a), resulting in a reduction in 430 the concentration of reactive sites for water molecules to bond 431 with (Figure 5c). The bulk of post-treated film remains similar 432 to that of the pure film, which explains the similarity in the 433 diffusion coefficients for both films (Figure 5b).

434 Finally, the chemical reaction between the diffusing 435 molecules and the substrate is described in our model by the forward $\left(k_{\mathrm{h}}\right)$ and backward $\left(k_{\mathrm{r}}\right)$ rate constants, which follows 436 Arrhenius' law

$$
k=A \mathrm{e}^{-E_{\mathrm{a}} / R_{\mathrm{g}} T}
$$

where $A$ is a pre-exponential factor, $R_{\mathrm{g}}$ is the universal gas 439 constant, and $E_{\mathrm{a}}$ is the activation energy.

Figure 6 illustrates how the logarithm of rate constants $441 \mathrm{f} 6$ changes with the inverse of temperature. The lines fit to 442 Arrhenius' law for the temperatures $26,37,50$, and $80^{\circ} \mathrm{C}$. The 443 EG treatment significantly affects the temperature dependence 444 of the rate constants. The differences between the slopes for 445 pure and treated films can be attributed to the morphological 446 reorganization taking place after the solvent treatment. The 447 values of $E_{\mathrm{a}}$ for the different film types are given in Table 1. For $448 \mathrm{t} 1$ both rate constants, the activation energies of EG-treated films 449 are higher than those for the pure film.

Table 1. Activation Energy Values for $k_{\mathrm{h}}$ and $k_{\mathrm{r}}$

\begin{tabular}{|cccc}
$E_{\mathrm{a}}$ for & pure & pretreated & post-treated \\
forward & 20948 & 67093 & 66384 \\
backward & 12057 & 19399 & 15302 \\
\hline
\end{tabular}

\section{CONCLUSIONS}

We have studied the absorption-desorption mechanism of 452 PEDOT:PSS and investigated the effects of EG treatment 453 (pretreatment and post-treatment). Gravimetric tests were 454 used to provide the evolution of water uptake in polymer films 455 at different temperatures and $\mathrm{RH}$ levels. Following the 456 pretreatment with EG, a morphological reorganization of the 457 polymer network and a loss in free volume resulted in a 458 decrease in water uptake. For the post-treated film, the removal 459 of the hydrophilic sites of PSS contributed to the reduction in 460 the amount of water reacting with the polymer.

The water transport process was described using a 462 diffusion-reaction model by implementing it in a COMSOL 463 Multiphysics and MATLAB optimization toolbox. A set of 464 parameters was identified to describe the kinetics of sorption 465 and desorption. The simulation results showed the same trend 466 as the one found in experimental tests for solubility change. 467 The evolution of each key parameter with respect to 468 temperature and solvent treatment was found consistent with 469 the physical considerations. 
471 This study highlights the dependence of the behavior of 472 PEDOT:PSS on the environmental conditions and the 473 influence of EG treatment on water transport kinetics. This 474 is the first step in the establishment of a complete Multiphysics 475 model needed for the design of complex PEDOT:PSS-based 476 components.

\section{ASSOCIATED CONTENT}

\section{S Supporting Information}

479 The Supporting Information is available free of charge at 480 https://pubs.acs.org/doi/10.1021/acsomega.9b02866.

481 Study about the validity of the Fickian diffusion model, 482 description of the diffusion-reaction model, and 483 database with all experimental results (PDF)

\section{AUTHOR INFORMATION}

\section{Corresponding Author}

486 *E-mail: gilles.lubineau@kaust.edu.sa. Phone: +966 (0) 1808 4872983.

488 ORCID

489 Gilles Lubineau: 0000-0002-7370-6093

\section{Author Contributions}

491 G.L. conceived the study. L.Z. and V.F. conducted the 492 experiments and simulation. All authors analyzed the results 493 and wrote the manuscript.

494 Notes

495 The authors declare no competing financial interest.

496 The datasets generated during and/or analyzed during the 497 current study are available from the corresponding author on 498 reasonable request.

\section{$499 \square$ ACKNOWLEDGMENTS}

500 The research reported in this publication was supported by the 501 funding from King Abdullah University of Science and 502 Technology (KAUST), under award number BAS/1/1315503 01-01.

\section{$504 \square$ REFERENCES}

505 (1) Cicoira, F.; Santato, C. Organic Electronics: Emerging Concepts 506 and Technologies, 3rd ed.; John Wiley \& Sons, 2013.

507 (2) Kim, N.; Kee, S.; Lee, S. H.; Lee, B. H.; Kahng, Y. H.; Jo, Y.-R.; 508 Kim, B.-J.; Lee, K. Highly conductive PEDOT:PSS nanofibrils 509 induced by solution-processed crystallization. Adv. Mater. 2014, 26, $5102268-2272$.

511 (3) Wu, F.; Li, P.; Sun, K.; Zhou, Y.; Chen, W.; Fu, J.; Li, M.; Lu, S.; 512 Wei, D.; Tang, X.; Zang, Z.; Sun, L.; Liu, X.; Ouyang, J. Conductivity 513 Enhancement of PEDOT:PSS via Addition of Chloroplatinic Acid 514 and Its Mechanism. Adv. Electron. Mater. 2017, 3, 1700047.

515 (4) Wang, C.; Sun, K.; Fu, J.; Chen, R.; Li, M.; Zang, Z.; Liu, X.; Li, 516 B.; Gong, H.; Ouyang, J. Enhancement of Conductivity and 517 Thermoelectric Property of PEDOT:PSS via Acid Doping and Single 518 Post-Treatment for Flexible Power Generator. Adv. Sustainable Syst. 519 2018, 2, 1800085.

520 (5) Okuzaki, H. Soft Actuators: Materials, Modeling, Applications, and 521 Future Perspectives; Asaka, K., Okuzaki, H., Eds.; Springer, 2014; 522 Chapter 8, pp 111-126.

523 (6) Zhang, S.; Cicoira, F. Water-Enabled Healing of Conducting 524 Polymer Films. Adv. Mater. 2017, 29, 1-6.

525 (7) Lin, P.; Yan, F.; Yu, J.; Chan, H. L. W.; Yang, M. The application 526 of organic electrochemical transistors in cell-based biosensors. Adv. 527 Mater. 2010, 22, 3655-3660.

528 (8) Nilsson, D.; Kugler, T.; Svensson, P. O.; Berggren, M. An all529 organic sensor-transistor based on a novel electrochemical transducer concept printed electrochemical sensors on paper. Sens. Actuators, B 530 2002, 86, 193-197.

(9) Hu, L.; Li, M.; Yang, K.; Xiong, Z.; Yang, B.; Wang, M.; Tang, 532 X.; Zang, Z.; Liu, X.; Li, B.; Xiao, Z.; Lu, S.; Gong, H.; Ouyang, J.; 533 Sun, K. PEDOT:PSS monolayers to enhance the hole extraction and 534 stability of perovskite solar cells. J. Mater. Chem. A 2018, 6, 16583- 535 16589 .

(10) Chen, R.; Sun, K.; Zhang, Q.; Zhou, Y.; Li, M.; Sun, Y.; Wu, Z.; 537 Wu, Y.; Li, X.; Xi, J.; Ma, C.; Zhang, Y.; Ouyang, J. Sequential 538 Solution Polymerization of Poly(3,4-ethylenedioxythiophene) Using 539 $\mathrm{V}_{2} \mathrm{O}_{5}$ as Oxidant for Flexible Touch Sensors. iScience 2019, 12, 66- 540 75.

(11) Okuzaki, H.; Suzuki, H.; Ito, T. Electrically driven PEDOT/ 542 PSS actuators. Synth. Met. 2009, 159, 2233-2236. 543

(12) Okuzaki, H.; Hosaka, K.; Suzuki, H.; Ito, T. Effect of 544 temperature on humido-sensitive conducting polymer actuators. Sens. 545 Actuators, A 2010, 157, 96-99.

(13) Okuzaki, H.; Kuwabara, T.; Kunugi, T. Theoretical study of 547 sorption-induced bending of polypyrrole films. J. Polym. Sci., Part B: 548 Polym. Phys. 1998, 36, 2237-2246.

(14) Okuzaki, H.; Kuwabara, T.; Kondo, T. Role and effect of 550 dopant ion on sorption-induced motion of polypyrrole films. J. Polym. 551 Sci., Part B: Polym. Phys. 1998, 36, 2635-2642.

(15) Okuzaki, H.; Kondo, T.; Kunugi, T. Characteristics of water in 553 polypyrrole films. Polymer 1999, 40, 995-1000.

554

(16) Lang, U.; Naujoks, N.; Dual, J. Mechanical characterization of 555 PEDOT:PSS thin films. Synth. Met. 2009, 159, 473-479. 556

(17) Kuş, M.; Okur, S. Electrical characterization of PEDOT:PSS 557 beyond humidity saturation. Sens. Actuators, B 2009, 143, 177-181. 558

(18) Sarkar, B.; Jaiswal, M.; Satapathy, D. K. Swelling kinetics and 559 electrical charge transport in PEDOT:PSS thin films exposed to water 560 vapor. J. Phys.: Condens. Matter. 2018, 30, 225101.

(19) El Yagoubi, J.; Lubineau, G.; Roger, F.; Verdu, J. A fully 562 coupled diffusion-reaction scheme for moisture sorption-desorption 563 in an anhydride-cured epoxy resin. Polymer 2012, 53, 5582-5595. 564

(20) Zhou, J.; Anjum, D. H.; Chen, L.; Xu, X.; Ventura, I. A.; Jiang, 565 L.; Lubineau, G. The temperature-dependent microstructure of 566 PEDOT/PSS films: insights from morphological, mechanical and 567 electrical analyses. J. Mater. Chem. C 2014, 2, 9903-9910. 568

(21) Ouyang, J.; Xu, Q.; Chu, C. W.; Yang, Y.; Li, G.; Shinar, J. On 569 the mechanism of conductivity enhancement in poly $(3,4-570$ ethylenedioxythiophene):poly(styrene sulfonate) film through solvent 571 treatment. Polymer 2004, 45, 8443-8450. 572

(22) Wei, Q.; Mukaida, M.; Naitoh, Y.; Ishida, T. Morphological 573 change and mobility enhancement in PEDOT:PSS by adding co- 574 solvents. Adv. Mater. 2013, 25, 2831-2836. 575

(23) Xia, Y.; Ouyang, J. Significant Conductivity Enhancement of 576 Conductive Poly (3,4-ethylenedioxythiophene):Poly- 577 (styrenesulfonate) Films through a Treatment with Organic 578 Carboxylic Acids and Inorganic Acids. ACS Appl. Mater. Interfaces 579 2010, 2, 474-483. 